\title{
Análisis ecológico de la violencia sexual de pareja en mujeres peruanas
}

\author{
Yordanis Enríquez-Canto; Yonathan Josué Ortiz-Montalvo; \\ Katherine Jenny Ortiz-Romaní; Giovani Martín Díaz-Gervasi
}

How to cite this article:

Enríquez-Canto, Y., Ortiz-Montalvo, Y.J., Ortiz-Romaní, K.J. \& Díaz-Gervasi, G.M. (2020). Ecological analysis of intimate partner sexual violence in Peruvian women. Acta Colombiana de Psicología, 23(1), $272-286$. doi: http://doi.org/10.14718/ACP.2020.23.1.13

Recibido, marzo 27/2019; Concepto de evaluación, mayo 10/2019; Aceptado, mayo 30/2019

\author{
Yordanis Enríquez-Canto* \\ Universidad Católica Sedes Sapientiae, Lima, Perú \\ ORCID: https://orcid.org/0000-0003-0449-5015 \\ Yonathan Josué Ortiz-Montalvo \\ Universidad Católica Sedes Sapientiae, Lima, Perú \\ ORCID: https://orcid.org/0000-0002-7678-8303 \\ Katherine Jenny Ortiz-Romaní \\ Universidad Católica Sedes Sapientiae, Lima, Perú \\ ORCID: https://orcid.org/0000-0003-0331-4446 \\ Giovani Martín Díaz-Gervasi \\ Universidad Católica Sedes Sapientiae, Lima, Perú \\ ORCID: https://orcid.org/0000-0002-2505-2689
}

\begin{abstract}
Resumen
Según el modelo ecológico, la violencia sexual de pareja se explica por la interacción de múltiples factores que coexisten en diversos niveles. Teniendo esto en cuenta, en el presente estudio se buscó medir la interacción existente entre los niveles según el aumento o disminución del riesgo de violencia sexual reciente por parte de la pareja actual en 21414 mujeres en edad fértil $-59.4 \%$ conviviente, $56 \%$ con trabajo independiente y $\mathrm{M}=30.3$ años $(\mathrm{DE}=9.2$ )—, por medio de un muestreo bietápico por conglomerados y estratificado por áreas departamentales del Perú. Para ello, se realizó un análisis de datos secundario a partir de lo obtenido en los módulos de violencia contra la mujer de la Encuesta Demográfica y de Salud Familiar (ENDES, 2017), con lo cual se determinaron cuatro modelos a partir de la regresión de Poisson y del cálculo de las razones de prevalencia y del área bajo la curva. Como resultado, se encontró que el $6.3 \%$ experimentó violencia sexual de pareja; que entre los factores individuales que aumentan el riesgo se incluyen, principalmente, los antecedentes de violencia familiar ( $\mathrm{PR}=1.19$; IC: 1.07-1.32); que en el microsistema se encuentran la violencia física (PR = 11.04 IC:8.49-14.36), las amenazas de tipo económico ( $\mathrm{PR}=2.58 \mathrm{IC}: 2.24-2.94)$ y la frecuencia de embriaguez del compañero $(\mathrm{PR}=1.98 \mathrm{IC}: 1.53-$ 2.56); mientras que la comunicación ( $\mathrm{PR}=0.45 \mathrm{IC}: 0.40-0.51)$ y pertenecer a quintiles superiores de riqueza $(\mathrm{PR}=0.59$ IC:0.45-0.76) resultaron ser factores protectores de la violencia sexual; y, por último, que la influencia simultánea y recíproca entre los niveles ecológicos no resultó paritaria respecto al peso predictivo de los factores.

Palabras clave: violencia sexual, violencia de pareja, modelo ecológico, factores de riesgo.
\end{abstract}

\footnotetext{
Facultad de Ciencias de la Salud, Esq. Constelaciones y Sol de Oro s/n, Urb., Sol de Oro, Los Olivos, Lima, Perú. C. P.: 15302. Tel.: +51(01) 533-0079, yenriquez@ucss.edu.pe
} 


\title{
Ecological analysis of intimate partner sexual violence in Peruvian women
}

Abstract

\begin{abstract}
The ecological model explains intimate partner sexual violence by the interaction of multiple factors that coexist at different levels. The aim of this paper was to analyze the interaction between those levels to estimate the increase or decrease of risk in recent sexual violence by the current partner in 21414 women at childbearing age $(\mathrm{M}=30.3$ years; $\mathrm{SD}=9.2)$, of whom $59.4 \%$ were in cohabitation and $56 \%$ had independent work. A two-stage sampling by conglomerates and stratified by areas of Peru's departments was used. To this end, a secondary data analysis from the Demographic and Family Health Survey (2017) was carried out taking into account the modules of violence against women. Four models were determined using the Poisson regression and calculating prevalence ratios and the area under the curve. The results showed that $6.3 \%$ of the sample experienced intimate partner sexual violence. Among the individual factors that increase the risk there is a history of domestic violence ( $\mathrm{PR}=1.19 \mathrm{IC}: 1.07-1.32)$. In the Microsystem, physical violence ( $\mathrm{PR}=11.04 \mathrm{IC}: 8.49-14.36)$, economic threats ( $P R$ $=2.58$ IC:2.24-2.94) and the partner's frequency of drunkenness were found ( $\mathrm{PR}=1.98 \mathrm{IC}: 1.53-2.56)$. On the contrary, communication ( $\mathrm{PR}=0.45 \mathrm{IC}: 0.40-0.51$ ) and belonging to higher wealth quintiles ( $\mathrm{PR}=0.59 \mathrm{IC}: 0.45-0.76)$ are protective factors of sexual violence. Finally, it is concluded that the simultaneous and reciprocal influence between ecological levels may not be equal regarding the predictive weight of the factors.

Key words: sexual violence, intimate partner violence, ecological model, risk factors.
\end{abstract}

\section{Introducción}

La violencia de pareja es un fenómeno de escala mundial que constituye tanto una vulneración de los derechos humanos como un fenómeno que tiene un impacto significativo en la salud de la mujer. La Organización Mundial de la Salud (OMS, 2002) la define como cualquier comportamiento por parte de un compañero íntimo dentro de una relación que cause daño psicológico, físico o sexual, y se ha encontrado que esta puede incluir uno o más actos de violencia física o sexual, además de que es uno de los tipos de violencia que con mayor frecuencia sufren las mujeres (OMS, 2012, 2013).

En particular, la violencia sexual de pareja se reporta cuando la mujer es forzada a tener relaciones o actos sexuales humillantes o degradantes (OMS, 2013), y se ha visto que esta puede aparecer tanto acompañada por otras formas de abuso como acontecer por sí sola, pero en ambos casos repercute en la salud con resultados adversos a corto y a largo plazo (Krebs, Breiding, Browne \& Warner, 2011; OMS, 2013), como se ha reportado con la angustia emocional, los pensamientos e intentos suicidas, la adicción a sustancias como el alcohol, y un mayor riesgo de adquirir infecciones de transmisión sexual (Devries et al., 2011; Garcia-Moreno, Jansen, Ellsberg, Heise \& Watts, 2006).

Con respecto a la prevalencia, a nivel internacional aproximadamente tres de cada diez mujeres han sufrido violencia física o sexual por parte de su compañero (OMS, 2013), cifra similar a la que se reporta en la región de las Américas, ya que esta alcanza el $29.8 \%$, siendo la más alta después de las regiones de África, Oriente Medio y Asia
Sudoriental (37\%)(OMS, 2013). Específicamente, en Perú, esta prevalencia en el curso de la vida de mujeres mayores de 15 años es del $22.5 \%$ (OMS, 2012), y, según lo reportado en la Encuesta Demográfica y de Salud familiar (ENDES) del 2017, la violencia sexual de pareja en los últimos doce meses es más frecuente en mujeres con una edad promedio de 35 años, separadas (19.8\%), con un mayor nivel educativo por parte del compañero $(7.3 \%)$, y pertenecientes al quintil inferior de riqueza (7.6\%) (INEI, 2017b).

Sin embargo, los datos sobre la prevalencia de la violencia sexual necesitan de profundización, razón por la cual se han realizado algunos estudios peruanos que indagan sobre los factores asociados a la violencia de pareja, aunque, como se ha encontrado, estos son escasos y a menudo la violencia sexual es pasada por alto. Como se encuentra en la literatura, algunos de dichos estudios se concentran en la violencia física (Blitchtein \& Reyes, 2012; Flake, 2005) y emplean índices que señalan la intensidad de la misma, pero sin distinguir entre los tipos de violencia (Castro, Cerellino \& Rivera, 2017), o consideran la violencia sexual en poblaciones específicas, como en mujeres embarazadas (Barrios et al., 2015) o residentes en comunidades de muy bajos ingresos (Nóblega, 2012), pero, a pesar de ello, los factores asociados a la violencia sexual reciente no se encuentran suficientemente explorados.

Ahora bien, una de las teorías que explica la alta prevalencia de la violencia de pareja es la teoría ecológica de Lory Heise (1998), en la cual la violencia de pareja se entiende a partir de su multicausalidad, según la visión del modelo ecológico de desarrollo humano de Urie Bronfenbrenner (1977). Este modelo considera al desarrollo humano como el 
resultado de la interacción constante entre el ser humano - que se encuentra en permanente actividad - y diversos factores ambientales cambiantes dentro de un proceso permanente de acomodación (Bronfenbrenner, 1977). De hecho, el término "ecológico" deriva del tener en cuenta al ser humano dentro de diferentes contextos en su entorno natural.

Heise (1998), específicamente, propone la teoría ecológica como una herramienta heurística para comprender la violencia contra la mujer a partir de distintos niveles - que se autocontienen-donde ocurre la interacción de factores personales, sociales y culturales que permite establecer variables predictoras en cada uno de los niveles (Heise, 1998). Igualmente, este modelo anidado permite tener en consideración las relaciones entre los predictores de la violencia en la pareja mediante un continuo que transita desde lo social a lo personal (OMS, 2002), lo que permite, a su vez, realizar distinciones precisas entre estos factores.

Este modelo ecológico de Heise (1998) fue adaptado al contexto peruano por parte de los autores del presente artículo (véase Figura 1), considerando los factores relacionados con las probabilidades de experimentar violencia sexual presentes en el exosistema, el microsistema y la historia personal de un grupo de mujeres, aunque no se consideraron factores relacionados con el macrosistema debido a que en la base de datos analizada — que surgió de la ENDES (2017) - no se recolectaron variables clasificables en dicho nivel del modelo ecológico, tal como se menciona en otras investigaciones (Devries et al., 2011; García-Moreno et al., 2006; Puente-Martínez, Ubillos-Landa, Echeburúa \& Páez-Rovira, 2016).
Como se puede observar, el exosistema abarca elementos sociales y comunitarios que engloban al microsistema y la historia personal (Heise, 1998), y, al respecto, diversos estudios sugieren que pertenecer a niveles socioeconómicos bajos aumentaría el riesgo de experimentar violencia sexual por parte de la pareja; que hay una relación inversa entre la violencia de pareja y el quintil de riqueza - tanto en un estudio con mujeres dominicanas (Bott, Guedes, Goodwin \& Mendoza, 2013) como en otro con mujeres mexicanas (Casique, 2010) —; que esta asociación se explicaría considerando la pobreza como un factor que alimenta el conflicto en las relaciones de pareja (OMS, 2002; Postmus, Plummer, McMahon, Murshid \& Kim, 2012), a la vez que favorece su aceptación (Casique, 2010); y que, a pesar de esta última asociación, otros estudios (Abramsky et al., 2011; Castillo, 2011) señalan ambigüedad con respecto a la relación entre la violencia sexual y la pertenencia de la mujer a un nivel socioeconómico.

Ahora bien, los factores del microsistema propios del contexto donde ocurre la violencia suelen estar conectados a una mayor probabilidad de violencia sexual, y, al respecto, un predictor para este problema en América Latina suele ser la frecuencia con la cual el compañero se embriaga (Casique, 2010; Jaén, Rivera, Amorin \& Rivera, 2015; Martínez, Landa, Echeburúa \& Rovira, 2016; Rey-Anacona, 2017); aspecto que ocurre también en el contexto peruano (Alarcón \& Ortiz, 2017; Blitchtein \& Reyes, 2012; Castro et al., 2017; Flake, 2005), en donde, según la información publicada por el INEI (2017b), alrededor del $50 \%$ de las mujeres peruanas experimentaron alguna vez violencia
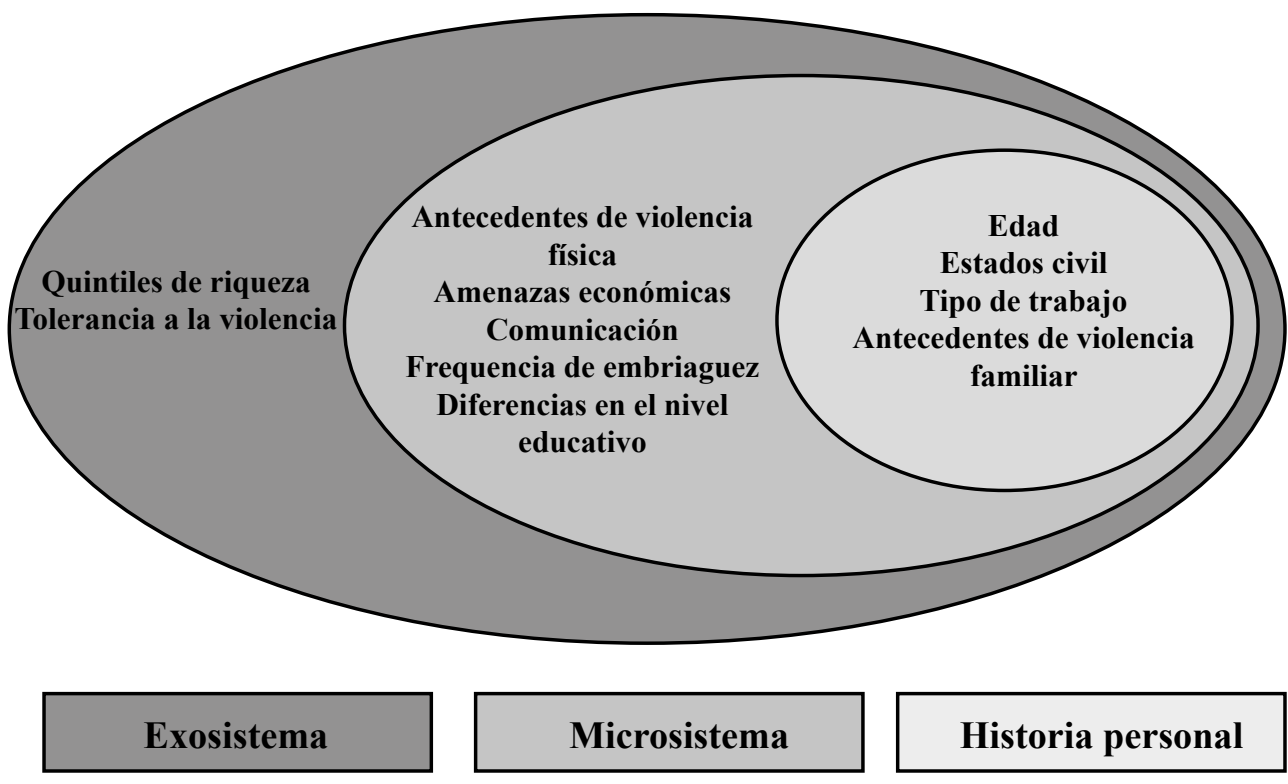

Microsistema

Historia personal

Figura 1. Clasificación de factores según el modelo ecológico, adaptado de Heise (1998). Fuente: elaboración propia. 
física de pareja cuando su compañero estaba ebrio, siendo el $69.8 \%$ de ellas de áreas rurales.

Igualmente, la violencia física ejercida por el compañero ha sido reportada como otro factor predictor, y, al respecto, se ha encontrado evidencia con datos provenientes tanto de Nicaragua (Ellsberg, Winkvist, Peña \& Stenlund, 2001) como de Uganda (Karamagi, Tumwine, Tylleskar \& Heggenhougen, 2006), España (Rodriguez-Menés, Puig \& Sobrino, 2014) y Estados Unidos (Krebs et al., 2011). Específicamente, en Perú (INEI, 2017b) se reportó casi un $31 \%$ de prevalencia de violencia física hacia la mujer, en donde los tipos de agresiones más reportados fueron los empujones o el lanzamiento de objetos (26\%), el ser abofeteadas $(18.2 \%)$ y haber sido golpeadas a puños (15.3\%). Respecto a esta asociación, la literatura señala una superposición de tipos de violencia (Krebs et al., 2011; Rodriguez-Menés et al., 2014), en donde las mujeres experimentan este tipo de violencia dentro de una misma relación o por parte de parejas distintas (García-Moreno et al., 2006).

Asimismo, recibir amenazas económicas también se ha relacionado con un mayor riesgo de experimentar violencia sexual (Postmus et al., 2012), y, al respecto, la prevalencia de este tipo de violencia, que puede incluir también las amenazas, va desde un $48 \%$ en Chile (Barría-Muñoz \& Macchiavello-Rodríguez, 2012) a un $29 \%$ en México (Casique, 2010). Específicamente, en Perú (INEI, 2017b) el $15.9 \%$ de las mujeres encuestadas reportó amenazas económicas por parte de su compañero. Cabe mencionar que tales amenazas son empleadas para intentar aislar a la mujer y así crear cierta dependencia y mantener un control coercitivo (Stark, 2007) hasta el punto en que las mujeres se ven forzadas a una dependencia económica y, por tanto, colocarlas en mayor riesgo de sufrir violencia sexual (Postmus et al., 2012). En Perú no existe información detallada sobre la prevalencia del abuso económico.

Por último, en el nivel de historia personal se encuentran los factores biológicos y experiencias aportadas a las relaciones interpersonales. Al respecto, estudios latinoamericanos señalan que tanto un historial de abuso infantil como el haber presenciado que el padre golpeara a la madre son predictores importantes de la misma (Casique, 2010; Jaén et al., 2015; Martínez et al., 2016; Rey-Anacona, 2017); y hallazgos en Perú confirman esto al corroborar el ciclo de violencia generacional familiar (Alarcón \& Ortiz, 2017; Aldarondo, Kantor \& Jasinski, 2002; Barrios et al. 2015; Blitchtein \& Reyes, 2012; Flake, 2005; Halford, Sanders \& Behrens, 2000).

Del mismo modo, la condición de empleo de la mujer también ha sido reportada como un factor de riesgo (Postmus et al., 2012), ya que, por una parte, en países europeos se ha señalado que las mujeres con empleo son más independientes y tienen más recursos para finalizar una relación violenta (Devries et al., 2011; García-Moreno et al., 2006); $y$, por otra, estudios en América Latina indican que estas mujeres se encuentran en mayor riesgo de experimentar violencia respecto a las que solo desempeñan labores en el hogar (Bott et al., 2013); asociación que ha sido confirmada por estudios peruanos (Castro et al., 2017; Flake, 2005).

Finalmente, cabe mencionar que las interacciones entre los factores presentes en los distintos niveles pueden modular la magnitud predictiva de la violencia de pareja (OMS, 2002), sin embargo, en la mayoría de los estudios peruanos sobre el tema no se examina la interacción dentro del modelo ecológico y, en general, se limitan a determinar el efecto predictivo de los factores de riesgo (Alarcón \& Ortiz, 2017; Barrios et al., 2015; Blitchtein \& Reyes, 2012; Castro et al., 2017; Nóblega, 2012), razón por la que resulta aún poco clara la acción de los diferentes niveles ecológicos al momento de establecer el efecto predictivo de los factores de riesgo de la violencia sexual reciente.

En síntesis, la violencia sexual de pareja es causada por una interacción simultánea y recíproca de múltiples factores que coexisten en los diversos niveles (Heise, 1998; OMS, 2002; Flake, 2005; Puente-Martínez et al., 2016). Por tanto, en la presente investigación empírica, que se realizó desde esta perspectiva ecológica, resulta insuficiente aislar el efecto de los factores de riesgo; por el contrario, se plantea que se debe indagar, adicionalmente, sobre los principales efectos causados por la interacción de los mismos. Esta visión permitió la emergencia de dos preguntas que guiaron la investigación, a saber: ¿qué factores tienen mayor poder explicativo en la violencia sexual? y ¿qué grupo de factores permanece como predictor relevante?

Así, teniendo en consideración los elementos planteados hasta este punto, en el presente trabajo se propuso, partiendo de la interacción entre los niveles ecológicos de la violencia, evaluar la influencia de los factores que intervienen en el aumento o disminución del riesgo de la violencia sexual reciente por parte de la pareja actual en mujeres peruanas.

\section{Método}

\section{Participantes}

Se empleó una muestra representativa de 21414 mujeres peruanas de 15 a 49 años de edad. Específicamente, se incluyeron las mujeres que en la encuesta ENDES de 2017 informaron tener pareja y que respondieron al módulo de violencia. De ellas, la media de edad del grupo fue de 30.3 años; el 10.88 \% residía en la región Lima metropolitana, el $30.72 \%$ en la región costa, el $32.51 \%$ en la región sierra y el $25.89 \%$ en la región selva; el $59.4 \%$ era conviviente; el 
$56 \%$ tenía un trabajo independiente; el $62.6 \%$ contó con un nivel de educación igual al de la pareja; y el $27.1 \%$ se encontraba en el quintil inferior de riqueza, mientras que el $9.7 \%$ se ubicaba en el quintil superior.

\section{Diseño}

Se realizó un estudio descriptivo transversal (Montero \& León, 2007) con un análisis secundario a partir de la base de datos de la encuesta ENDES de 2017, realizada de manera representativa a nivel nacional por el Instituto Nacional de Estadística e Informática (INEI, 2017a). El muestreo fue bietápico por conglomerados - unidades primarias de muestreo-, compuesto por 140 viviendas aproximadamente, y estratificado por áreas urbanas y zonas rurales —unidades secundarias de muestreo- de los 24 departamentos y dos provincias del Perú (INEI, 2017a). Considerando la complejidad del muestreo para determinar los errores de estimación, se emplearon diversas variables, consultables en el apéndice B del informe ENDES (INEI, 2017a), en donde se señala el estimador utilizado y la población de referencia. Considérese como ejemplo para el cálculo de los errores de muestreo de la población total la variable "actualmente unida", una proporción con una población de base de mujeres de 15 a 49 años que arrojó un valor estimado de $0.566(56.6 \%)$, con un error estándar de $0.005(0.5 \%)$ y un intervalo de $95 \%$ de confianza (IC $95 \%=0.555-0.577$ ).

\section{Instrumento}

Se hizo uso de los datos obtenidos en la Encuesta Demográfica y de Salud Familiar (ENDES) de 2017, específicamente en su sección 10, que consta de 48 preguntas relacionadas con actos de violencia física y sexual tanto a lo largo de la vida de la participante como en los últimos 12 meses. El tiempo aproximado de respuesta para esta sección fue de 30 minutos. En particular, en esta se obtiene información sobre las consecuencias de los episodios de violencia y la posterior asistencia a servicios de salud.

Inicialmente, en la medición de la violencia se utilizó el enfoque de umbral de una sola pregunta con el fin de determinar si la encuestada había experimentado violencia o no. Así, a las participantes que respondieron afirmativamente se les realizó un conjunto de preguntas sobre las características de la misma (p. ej., frecuencia). Para examinar la prevalencia de violencia y sus correlatos, la ENDES sigue el modelo y metodología de las encuestas demográficas y de salud de Kishor y Kiersten (2006).

En Perú, este cuestionario fue aplicado por primera vez en el año 2000, y posteriormente se ha empleado anualmente, con un diseño de encuesta continua, para un período de cinco años (2004-2008) y luego de tres años
(2009-2011; 2012-2014; 2015-2017). Por otra parte, este instrumento refleja la metodología comparativa de medición del estudio multipaís de la OMS sobre salud de la mujer y violencia doméstica (OMS, 2005), lo que lo convierte en herramienta adecuada para medir la prevalencia de violencia y sus características contextuales. Sin embargo, cabe mencionar que a pesar de su uso repetido y difundido, sus datos psicométricos no se encuentran disponibles.

En lo que respecta a la sección 10, se utilizaron nueve preguntas relacionadas con la violencia sexual de pareja, las cuales, teniendo como variable principal la violencia sexual de pareja — definida por la OMS (2013) como relaciones sexuales forzadas y otras formas de coerción sexual dentro de una relación íntima-, se construyeron a partir de dos preguntas: (a) ¿su (último) esposo (compañero) alguna vez ha utilizado la fuerza física para obligarla a tener relaciones sexuales aunque usted no quería en los últimos 12 meses?, y (b) ¿su (último) esposo (compañero) alguna vez la obligó a realizar actos sexuales que usted no aprueba en los últimos 12 meses?, que se respondían de manera dicotómica (sí o no) —en estas dos preguntas se obtuvo un alfa de Cronbach de .72-.

Las siete preguntas restantes siguen el modelo teórico de Heise (1998) y se agrupan en tres grupos de factores - no se consideraron los factores del macrosistema debido a que en esta encuesta no se recolectaron variables clasificables en este nivel del modelo ecológico-:

1. Factores individuales. Edad de la mujer (en años); estado civil (casadas, conviviente, separadas) - las categorías viudas y divorciadas se excluyeron del análisis porque constituían menos del $1 \%$ de la muestra-; tipo de trabajo (dependiente, independiente); y antecedentes de violencia física del padre contra la madre (“ ¿su papá le pegó alguna vez a su mamá?”, y “'desde que usted tenía 15 años de edad alguna vez otra persona aparte de su esposo/compañero le pegó, la pateó, abofeteó o maltrató físicamente?", con respuestas de "sí" o "no").

2. Factores del microsistema. Violencia física por la pareja actual (" $i s u$ [último] esposo [compañero] alguna vez la sacudió, la abofeteó, la golpeó con el puño, la ha pateado, la trató de estrangular, la amenazó o la agredió con un cuchillo, pistola u otro tipo de arma en los últimos 12 meses?", con respuestas de "si'" o "no" y un alfa de Cronbach de .81); amenaza de la pareja con quitarle la ayuda económica ("¿la ha amenazado con quitarle la ayuda económica?", con respuestas de "sí" o "no"); comunicación con la pareja actual (con preguntas sobre si él es cariñoso, si pasa el tiempo libre con ella, si le consulta su opinión en diferentes temas del hogar y si respeta sus deseos y derechos, con respuestas de "sí" o "no" y un alfa de Cronbach de .77); 
frecuencia de embriaguez (" $¿$ su esposo [compañero] se embriaga con mucha frecuencia, algunas veces o nunca?", con opciones de respuesta de "no se embriaga", "algunas veces se embriaga" y "se embriaga seguido"); y diferencia respecto al nivel educativo de la pareja — definida como el nivel máximo en la formación educativa alcanzado con respecto al hombre- (con opciones de respuesta de "igual", "ella más que él" y "él más que ella").

3. Factores del exosistema. Se consideraron los quintiles de riqueza, definidos en términos de riqueza en los hogares encuestados (cinco quintiles respecto a activos, en lugar de ingresos o consumos [quintil inferior, segundo quintil, quintil intermedio, cuarto quintil, quintil superior]), según Enríquez-Canto, Ortiz-Romaní y Ortiz-Montalvo (2017); y la tolerancia a la violencia ("en su opinión, ¿usted está de acuerdo que él golpee a su esposa cuando sale sin decirle nada, descuida a los niños, discute con él, se niega a tener relaciones sexuales o si quema la comida?", con respuestas de "sí" o "no" y un alfa de Cronbach de .80).

\section{Procedimiento}

Se aplicaron los módulos de la ENDES en cada uno de los departamentos del Perú entre los meses de febrero y diciembre de 2017, tras contactar a las mujeres residentes en las viviendas seleccionadas de manera aleatoria y pertenecientes a un conglomerado previamente determinado al azar. Para tal fin, una encuestadora entrenada recolectó la información mediante entrevistas cara a cara, asistida por una computadora personal en el hogar de las encuestadas. Al inicio de la entrevista se leyó el consentimiento informado y se obtuvo el asentimiento verbal. La recolección de datos se dio acorde a la disponibilidad de las participantes, con la instrucción de interrumpir la entrevista en ausencia de privacidad.

\section{Consideraciones éticas}

Con el fin de maximizar la seguridad de los datos recopilados y la no divulgación de información personal, el INEI realizó una capacitación especializada para encuestadores, se entrevistó a una sola mujer por hogar y se mantuvo la completa privacidad durante la entrevista.

\section{Análisis de los datos}

Se calcularon las medias y desviaciones estándar de las variables cuantitativas, así como las frecuencias y los porcentajes para las variables cualitativas con el programa estadístico Stata SE 14. Inicialmente, se comprobó con la prueba de Kolmogorov-Smirnov que las variables numéricas se ajustaran a una distribución normal para la edad $(p>.05)$ en los grupos de comparación. Posteriormente, se verificó la homogeneidad de varianzas (Prueba de Levene), $\mathrm{y}$, comprobados estos requisitos, se realizaron las pruebas t de Student y el Chi-cuadrado para el análisis bivariado descriptivo (edad). La tendencia a la colinealidad se evaluó mediante el factor de inflación de la varianza, y en el análisis multivariado se empleó la regresión de Poisson con la estimación de la varianza robusta y la razón de prevalencia (PR) (Espelt A, Marí-Dell'Olmo, Penelo, Bosque-Prous, 2017), con intervalos de confianza al $95 \%$, considerando un nivel de significancia estadística menor o igual a 0.05 .

Finalmente, se determinaron cuatro modelos, cuyos coeficientes representan el aumento o la disminución de la probabilidad de violencia sexual reciente asociada con el cambio de una unidad (o categoría) en una variable independiente. El primer modelo determina la probabilidad de violencia sexual reciente considerando los factores de la historia personal; en el segundo se midieron los efectos de los factores del microsistema; el tercero midió la probabilidad de violencia sexual considerando los factores del exosistema; y el cuarto consideró los factores de los tres niveles ecológicos para comprender el efecto de la interacción de estos en la violencia sexual. Para terminar, se estimó la bondad de ajuste con el criterio de información de Akaike (AIC), y se calculó el área bajo la curva (AUC) de cada modelo estimado.

\section{Resultados}

En la Tabla 1 se describen los factores conectados a la violencia sexual reciente en Perú. En general, del total de la muestra, el $6.3 \%$ sufrió violencia sexual por su pareja en los últimos doce meses de aplicada la encuesta, el $29.5 \%$ sufrió violencia física, el $43.6 \%$ presentó antecedentes de violencia del padre a la madre, casi un $15 \%$ fue amenazada económicamente, y el $73.1 \%$ de los compañeros se embriagaba algunas veces.

Por otra parte, se reportó una asociación estadísticamente significativa entre el estado civil de las mujeres y la violencia sexual por parte de la pareja $(p=.001)$; casi el $20 \%$ de las mujeres separadas sufrió violencia sexual en los últimos doce meses; se confirmó una asociación entre la edad de las mujeres y la violencia sexual ( $p=.001)$; las mujeres que experimentaron violencia contaban con una media de edad superior $(\mathrm{M}=34.80 ; \mathrm{DE}= \pm 8.08)$ respecto a aquellas que no lo hicieron $(\mathrm{M}=32.14 ; \mathrm{DE}= \pm 7.88)$; se reportó asociación entre los antecedentes de violencia del padre hacia la madre y la violencia sexual $(p=.001)$; y el $8 \%$ de las mujeres que experimentaron violencia sexual evidenció antecedentes de violencia del padre hacia la madre. 
Tabla 1.

Violencia sexual reciente por la pareja actual y datos sociodemográficos

\begin{tabular}{|c|c|c|}
\hline & $\mathrm{n}$ & $\%$ \\
\hline \multicolumn{3}{|c|}{ Violencia sexual } \\
\hline No & 20073 & 93.74 \\
\hline Sí & 1341 & 6.26 \\
\hline \multicolumn{3}{|c|}{ Factores individuales } \\
\hline \multicolumn{3}{|l|}{ Edad } \\
\hline Edad de la mujer (media $\pm D E$ ) & \multicolumn{2}{|c|}{$30.35 \pm 9.22$} \\
\hline \multicolumn{3}{|l|}{ Estado civil } \\
\hline Casadas & 5790 & 27.04 \\
\hline Conviviente & 13004 & 60.73 \\
\hline Separadas & 2499 & 11.67 \\
\hline Viudas & 65 & 0.30 \\
\hline Divorciadas & 56 & 0.26 \\
\hline \multicolumn{3}{|l|}{ Tipo de trabajo } \\
\hline Dependiente & 6644 & 43.29 \\
\hline Independiente & 8703 & 56.71 \\
\hline \multicolumn{3}{|c|}{ Antecedente de agresión fisicica de su padre a su madre } \\
\hline No & 11635 & 56.34 \\
\hline Sí & 9018 & 43.66 \\
\hline \multicolumn{3}{|c|}{ Antecedente de agresión fisica del padre a la mujer } \\
\hline No & 20389 & 95.24 \\
\hline Sí & 1018 & 4.76 \\
\hline \multicolumn{3}{|c|}{ Factores del microsistema } \\
\hline \multicolumn{3}{|l|}{ Violencia fisica por pareja actual } \\
\hline No & 15099 & 70.51 \\
\hline Sí & 6315 & 29.49 \\
\hline \multicolumn{3}{|l|}{ Amenaza económica } \\
\hline No & 18222 & 85.09 \\
\hline Sí & 3192 & 14.91 \\
\hline \multicolumn{3}{|l|}{ Comunicación con la pareja } \\
\hline No & 1030 & 4.81 \\
\hline Sí & 20384 & 95.19 \\
\hline \multicolumn{3}{|l|}{ Frecuencia de embriaguez } \\
\hline No se embriaga & 3419 & 19.73 \\
\hline Algunas veces se embriaga & 12668 & 73.12 \\
\hline Se embriaga seguido & 1238 & 7.15 \\
\hline \multicolumn{3}{|c|}{ Diferencia en el nivel educativo con la pareja } \\
\hline Ambos con el mismo nivel & 13480 & 62.95 \\
\hline Mujer con mayor nivel & 3036 & 14.18 \\
\hline Hombre con mayor nivel & 4898 & 22.87 \\
\hline \multicolumn{3}{|c|}{ Factores del exosistema } \\
\hline \multicolumn{3}{|l|}{ Quintiles de riqueza } \\
\hline Quintil inferior & 5818 & 27.17 \\
\hline Segundo quintil & 5974 & 27.90 \\
\hline Quintil intermedio & 4335 & 20.24 \\
\hline Cuarto quintil & 3192 & 14.91 \\
\hline Quintil superior & 2095 & 9.78 \\
\hline \multicolumn{3}{|l|}{ Tolerancia social a la violencia } \\
\hline No & 20930 & 98.11 \\
\hline Sí & 404 & 1.89 \\
\hline
\end{tabular}


Asimismo, se encontró una asociación significativa entre la violencia sexual y la comunicación con la pareja ( $p=.001)$; el $42.5 \%$ de las mujeres que reportaron violencia sexual expresaron ausencia de comunicación con su pareja; se obtuvo una asociación entre la violencia física hacia la mujer y la violencia sexual por parte de la pareja $(p=.001)$; el $19 \%$ de las mujeres que experimentaron violencia física igualmente sufrieron violencia sexual; existe asociación entre la frecuencia de embriaguez y la violencia sexual reciente $(p=.001)$; y el $32 \%$ de las mujeres que experimentaron violencia sexual señaló que su pareja se embriagaba seguido. El resto de los factores estudiados se puede observar en la Tabla 2.

Con respecto a la regresión múltiple realizada, el Modelo 1 estimó la probabilidad de violencia sexual reciente a partir de los factores personales (véase Tabla 3 ).

Tabla 2.

Análisis bivariado entre los factores y violencia sexual reciente por parte de la pareja

\begin{tabular}{|c|c|c|c|c|c|}
\hline & \multicolumn{2}{|c|}{ Violencia sexual } & \multirow[b]{2}{*}{$\mathrm{p}$} & \multirow[b]{2}{*}{ PR (IC $95 \%)$} & \multirow[b]{2}{*}{$\mathrm{p}$} \\
\hline & $\begin{array}{c}\text { No } \\
\mathrm{n}(\%)\end{array}$ & $\begin{array}{c}\text { Sí } \\
\text { n (\%) }\end{array}$ & & & \\
\hline \multicolumn{6}{|c|}{ Factores individuales } \\
\hline \multicolumn{6}{|l|}{ Edad } \\
\hline Edad de la mujer (media $\pm D E$ ) & $32.14 \pm 7.88$ & $34.80 \pm 8.08$ & $<.001$ & $1.04(1.03-1.05)$ & $<.001$ \\
\hline Estado civil & & & $<.001$ & & \\
\hline Casadas & $5516(95.27)$ & $274(4.73)$ & & Referencia & - \\
\hline Conviviente & $12453(95.76)$ & $551(4.24)$ & & $0.89(0.77-1.03)$ & 0.126 \\
\hline Separadas & $2005(80.23)$ & $494(19.77)$ & & $4.18(3.63-4.80)$ & $<.001$ \\
\hline Tipo de trabajo & & & .791 & & \\
\hline Dependiente & $6141(92.43)$ & $503(7.57)$ & & Referencia & - \\
\hline Independiente & $8054(92.54)$ & $649(7.46)$ & & $0.98(0.88-1.10)$ & .791 \\
\hline \multicolumn{3}{|c|}{ Antecedente de agresión fisica de su padre a su madre } & $<.001$ & & \\
\hline No & $11083(95.26)$ & $552(4.74)$ & & Referencia & - \\
\hline Sí & $8294(91.97)$ & $724(8.03)$ & & $1.69(1.52-1.88)$ & $<.001$ \\
\hline \multicolumn{3}{|c|}{ Antecedente de agresión física del padre a la mujer } & $<.001$ & & \\
\hline No & $19160(93.97)$ & $1229(6.03)$ & & Referencia & - \\
\hline Sí & $907(89.10)$ & $111(10.90)$ & & $1.80(1.50-2.17)$ & $<.001$ \\
\hline \multicolumn{6}{|c|}{ Factores del microsistema } \\
\hline Violencia fisica por pareja actual & & & $<.001$ & & \\
\hline No & $14988(99.26)$ & $111(0.74)$ & & Referencia & - \\
\hline Sí & $5085(80.52)$ & $1230(19.48)$ & & $26.49(21.86-32.10)$ & $<.001$ \\
\hline Amenaza económica & & & $<.001$ & & \\
\hline No & $17749(97.40)$ & $473(2.60)$ & & Referencia & - \\
\hline Sí & $2324(72.81)$ & $868(27.19)$ & & $10.47(9.42-11.64)$ & $<.001$ \\
\hline Comunicación con la pareja & & & $<.001$ & & \\
\hline No & $592(57.48)$ & $438(42.52)$ & & Referencia & - \\
\hline Sí & $19481(95.57)$ & $903(4.43)$ & & $0.10(0.09-0.11)$ & $<.001$ \\
\hline Frecuencia de embriaguez & & & $<.001$ & & \\
\hline No se embriaga & $3332(97.46)$ & $87(2.54)$ & & Referencia & - \\
\hline Algunas veces se embriaga & $11961(94.42)$ & $707(5.58)$ & & $2.19(1.76-2.73)$ & $<.001$ \\
\hline Se embriaga seguido & $841(67.93)$ & $397(32.07)$ & & $12.60(10.08-15.74)$ & $<.001$ \\
\hline \multicolumn{3}{|l|}{ Diferencia en el nivel educativo con la pareja } & .005 & & \\
\hline Ambos con el mismo nivel & $12690(94.14)$ & $790(5.86)$ & & Referencia & - \\
\hline Mujer con mayor nivel & $2816(92.75)$ & $220(7.25)$ & & $1.24(1.07-1.43)$ & .004 \\
\hline Hombre con mayor nivel & $4567(93.24)$ & $331(6.76)$ & & $1.15(1.01-1.30)$ & .024 \\
\hline \multicolumn{6}{|l|}{ Ouintiles de riaueza } \\
\hline Quintiles de riqueza & & & $<.001$ & & \\
\hline Quintil inferior & $5374(92.37)$ & $444(7.63)$ & & Referencia & - \\
\hline Segundo quintil & $5550(92.90)$ & $424(7.10)$ & & $0.93(0.82-1.05)$ & .267 \\
\hline Quintil intermedio & $4076(94.03)$ & $259(5.97)$ & & $0.78(0.67-0.90)$ & .001 \\
\hline Cuarto quintil & $3048(95.49)$ & $144(4.51)$ & & $0.59(0.49-0.71)$ & $<.001$ \\
\hline Quintil superior & $2025(96.66)$ & $70(3.34)$ & & $0.44(0.34-0.56)$ & $<.001$ \\
\hline Tolerancia social a la violencia & & & .721 & & \\
\hline No & $19622(93.75)$ & $1308(6.25)$ & & Referencia & - \\
\hline Sí & $377(93.32)$ & $27(6.68)$ & & $1.06(0.74-1.54)$ & .721 \\
\hline
\end{tabular}


Acá, la edad resultó estar asociada positivamente con la violencia ( $\mathrm{PR}=1.03$; IC: $1.02-1.04 ; p=.001)$; las mujeres con antecedentes de violencia de parte del padre hacia la madre presentaron más probabilidades de violencia sexual por el compañero ( $\mathrm{PR}=1.53$; IC: $1.36-1.72 ; p=.001)$ respecto a las que no reportaron dicho antecedente; y quienes señalaron agresión de parte del padre mostraron más riesgo de violencia sexual reciente $(\mathrm{PR}=1.61$; IC: $1.32-1.96$; $p=.001)$ respecto a las que no la reportaron. En lo que respecta al AIC, el modelo reportó un valor de 7637.

Con el Modelo 2 se evaluaron las relaciones entre los factores del microsistema y la probabilidad de experimentar violencia (véase la Tabla 3). Acá, la violencia física de pareja resultó ser el predictor más fuerte del modelo; las mujeres que han experimentado violencia física por parte de su pareja presentaron mayor probabilidad de sufrir violencia sexual ( $\mathrm{PR}=12.8$; IC: $10.15-16.24 ; p=.001)$ que aquellas que no; las mujeres que recibieron amenazas económicas ( $\mathrm{PR}=2.6$; IC: $2.29-2.95 ; p=.001)$ tuvieron más probabilidad de violencia sexual que aquellas que no fueron amenazadas; las encuestadas que señalaron que su pareja se embriaga seguido mostraron el doble de riesgo de violencia sexual reciente $(\mathrm{PR}=2.01$; IC: $1.61-2.51$; $p=0.001$ ) respecto a las que reportaron que su pareja se embriaga solo algunas veces; y las mujeres que manifestaron comunicación con su compañero presentaron menos probabilidades de experimentar violencia sexual respecto a aquellas que declararon ausencia de comunicación $(\mathrm{PR}=0.44$
IC: $0.39-0.49 ; p=.001)$. En el AIC, este modelo reportó un valor de 5656 .

Por otra parte, con el Modelo 3 se consideró la relación entre los factores del exosistema y la violencia sexual en los últimos doce meses, y aquí se encontró una relación inversa entre la pertenencia al quintil y la violencia; y las mujeres pertenecientes al quintil superior demostraron menor probabilidad de presentar violencia sexual $(\mathrm{PR}=0.44$; IC: $0.34-0.56 ; p=.001$ ) respecto a las del quintil inferior. Este modelo reportó el valor de AIC más alto: 9957 (véase Tabla 3).

Finalmente, en el Modelo 4 se evaluó la interacción conjunta de los factores presentes en cada nivel con la violencia sexual. Como se puede observar, en la Figura 2 se evidencia la interconexión entre los niveles ecológicos, donde la violencia física de la pareja permanece como fuerte predictor, seguida por las amenazas económicas; el efecto de los antecedentes de violencia familiar es atenuado, pues vivenciar violencia del padre a la madre disminuye de $1.53(p=.001)$ a 1.19 $(p=.001)$; y el efecto protector del quintil de riqueza superior disminuye de $0.44(p=.001)$ a $0.59(p=.001)$. Este modelo reportó el valor de AIC más bajo: 4380.

Para finalizar, como último paso se estimó la capacidad discriminante de cada modelo con el AUC, y los puntajes resultantes para el Modelo 1 fueron de 0.61 (IC $95 \%=0.59-0.63$ ), de 0.89 (IC $95 \%=0.88-0.90$ ) para el Modelo 2, de 0.58 (IC $95 \%=0.56-0.60$ ) para el Modelo 3 , y de 0.90 (IC $95 \%=0.89-0.91$ ) para el Modelo 4.

\section{Discusión}

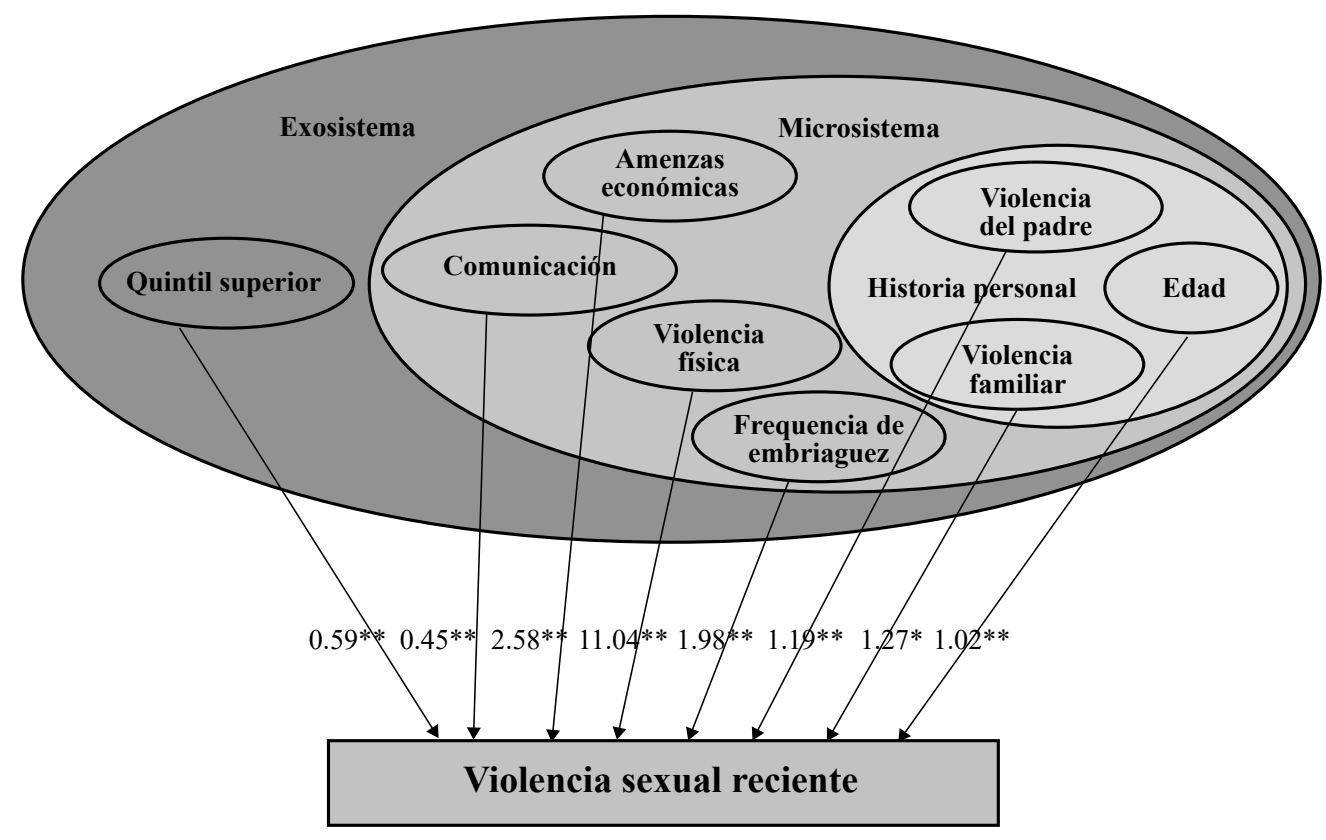

Figura 2. Factores asociados a la violencia sexual reciente según los niveles del modelo ecológico adaptado. Los valores son coeficientes de regresión del Modelo 4. ${ }^{*} \mathrm{p}<.05 ;{ }^{* *} \mathrm{p}<.001$. Fuente: elaboración propia. 


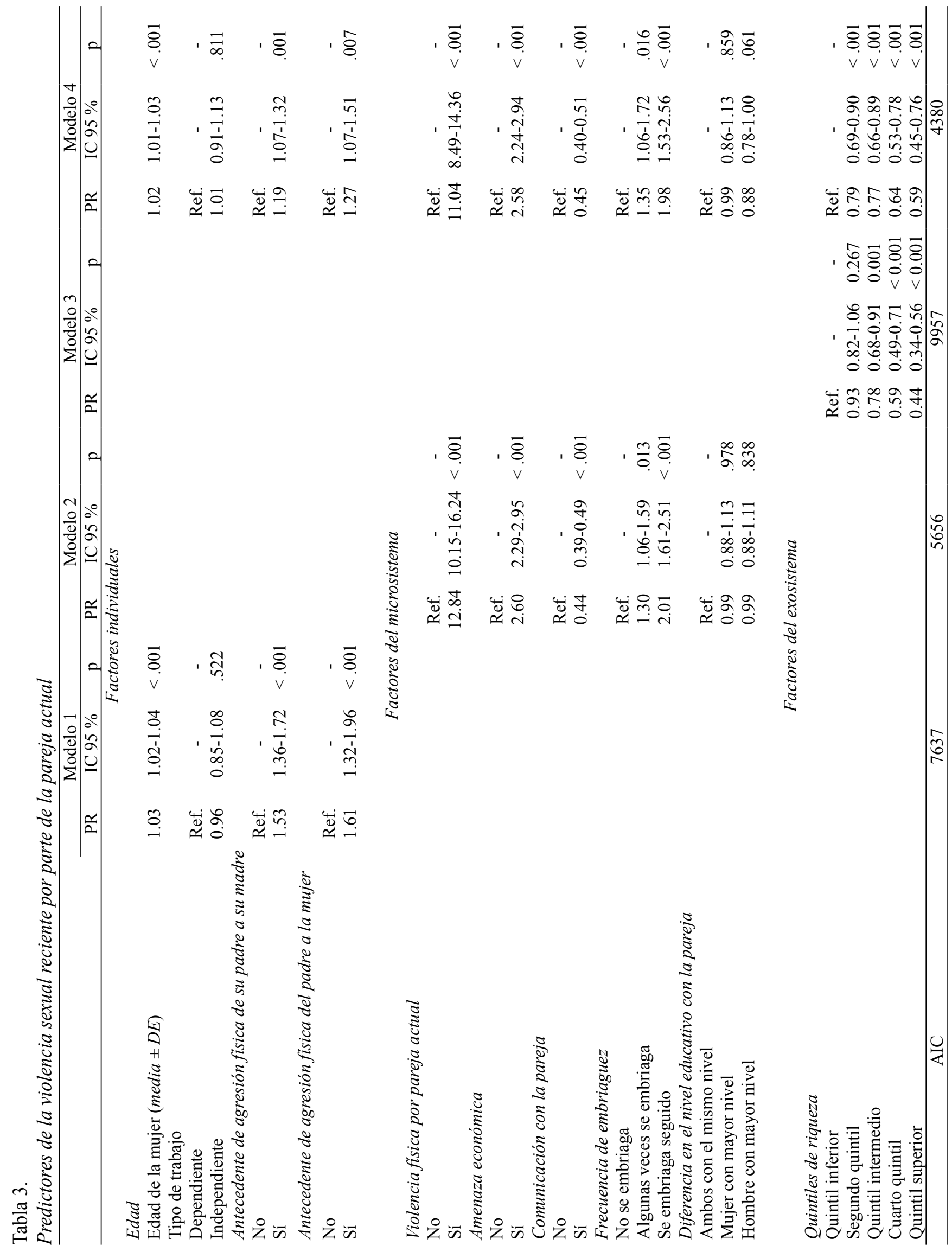


Desde la perspectiva del modelo ecológico, la violencia sexual de pareja se explica por la interacción de múltiples factores que coexisten en los diversos niveles del modelo (Heise, 1998; OMS, 2002); razón por la cual la investigación empírica en violencia debería indagar sobre los efectos causados por la interacción de dichos niveles ecológicos. Teniendo esto en cuenta, en la presente investigación se propuso como objetivo analizar la interacción entre estos niveles en el aumento o disminución del riesgo de violencia sexual reciente por parte de la pareja actual en mujeres peruanas.

Como resultados generales, entre los factores personales se encontró que las mujeres que vivenciaron episodios de violencia familiar tienen más probabilidades de experimentar violencia sexual reciente. Al respecto, los antecedentes intrafamiliares de violencia han sido documentados en la literatura como un factor asociado al riesgo de violencia sexual de pareja en países como Colombia (Martínez et al., 2016; Rey-Anacona, 2017), México (Casique, 2010; Jaén et al., 2015) y Perú (Alarcón \& Ortiz, 2017; Blitchtein \& Reyes, 2012; Flake, 2005). Asimismo, en el presente estudio cuatro de cada diez peruanas vivenciaron episodios de violencia física intrafamiliar, y un historial de violencia en la infancia resultó predictor de comportamientos violentos, lo cual es consistente con el ciclo de violencia generacional transmitido en las familias que se reporta en distintas investigaciones (Aldarondo et al., 2002; Halford et al., 2000). De hecho, desde la teoría del aprendizaje social (Bandura, 1977) se explica que los episodios de violencia entre los padres reforzarían lo habitual de la violencia en las relaciones de pareja, conduciendo a una mayor probabilidad de experimentarla en el futuro (Halford et al., 2000).

Con respecto al nivel del microsistema, destaca el rol de la violencia física en la violencia sexual reciente, siendo la primera una fuerte predictora de la segunda. Así, las mujeres que sufrieron violencia física por su pareja presentaron más probabilidades de ser violentadas sexualmente. Al respecto, estudios a nivel internacional reportaron hallazgos similares en España (Rodriguez-Menés et al., 2014), Estados Unidos (Krebs et al., 2011), Uganda (Karamagi et al., 2006) y Nicaragua (Ellsberg et al., 2001), con lo que se confirma que, a pesar de la diversidad cultural, la violencia física suele ir acompañada de la violencia sexual. Asimismo, la literatura señala una superposición de tipos de violencia de pareja (Krebs et al., 2011; Rodriguez-Menés et al., 2014), $\mathrm{y}$, de hecho, se ha encontrado que es posible experimentar diferentes tipos de violencia dentro de una misma relación o a lo largo de la vida de la mujer por parte de distintas parejas y en contextos diversos (García-Moreno et al., 2006). Sumado a lo anterior, un episodio de violencia de pareja puede implicar una combinación de amenazas, actos de agresión física y violencia sexual (Krebs et al., 2011), $\mathrm{e}$, incluso, la violencia sexual puede ser parte del castigo que inflige el compañero a la mujer (Kamaragi et al., 2006), pues en ocasiones en caso de que una mujer afirme que no quiere tener actividad sexual, el compañero puede golpearla y luego obligarla a tener relaciones sexuales. Esta información ha sido confirmada por el estudio comparativo latinoamericano de Bott et al. (2013).

Otro hallazgo interesante revela que las mujeres que reciben amenazas económicas tienen más probabilidad de experimentar violencia sexual. Al respecto, Postmus et al. (2012) proponen que no se ha explorado suficientemente de manera empírica la relación entre la violencia económica, que puede incluir estas amenazas, y otras formas de violencia de pareja; pero, según la teoría del control coercitivo de Stark (2007), un agresor intenta establecer posiciones de poder mediante diversas tácticas, como las amenazas y los actos de violencia sexual, que permiten mantenerlo, de modo que las mujeres que se ven forzadas a una dependencia económica se encuentran en mayor riesgo de sufrir violencia sexual y de no abandonar la relación. En Latinoamérica, un estudio chileno señaló un $48 \%$ de prevalencia de uso de violencia económica (Barría-Muñoz \& Macchiavello-Rodríguez, 2012), comparado con un 29.3\% en mujeres mexicanas (Casique, 2010). Por su parte, los hallazgos del presente estudio arrojan un $15 \%$ del total de la muestra que experimentó amenazas económicas. Estas diferencias se podrían explicar por el poco espacio dedicado en la ENDES a las preguntas que indagan sobre dicho tipo de violencia.

Por otra parte, los resultados confirman que las mujeres que tienen parejas que se embriagan con mayor frecuencia tienen más posibilidades de experimentar violencia sexual reciente. Con respecto a este aspecto, otros estudios reportan hallazgos similares tanto en Perú (Alarcón \& Ortiz, 2017; Blitchtein \& Reyes, 2012; Castro et al., 2017; Flake; 2005) como a nivel internacional (Abramsky et al., 2011; Devries et al., 2011). En Perú, específicamente, el consumo frecuente de alcohol está asociado a la imagen de masculinidad y al rol social atribuido (Castro et al., 2017), y, al respecto, los datos del estudio revelan que siete de cada diez peruanas reportan que su compañero se embriaga algunas veces. Este dato es preocupante, ya que el consumo de bebidas alcohólicas ha estado tradicionalmente relacionado con la violencia y con las conductas agresivas (Puente-Martínez et al., 2016). Sin embargo, cabe mencionar que el consumo de alcohol en un episodio de maltrato no necesariamente significa que sea la causa de la violencia, y es importante considerar que la violencia de pareja ocurre frecuentemente 
sin el consumo de alcohol, a pesar de que pueda tener un efecto desinhibitorio en el comportamiento (Abramsky et al., 2011; Alarcón \& Ortiz, 2017).

Ahora bien, entre los factores protectores, los resultados indican la importancia de la comunicación en la pareja, ya que las mujeres que manifestaron mantener comunicación con su compañero presentaron menor probabilidad de experimentar violencia sexual. En ese mismo aspecto, otro estudio peruano confirma este hallazgo (Blitchtein \& Reyes, 2012), y es importante considerar que la violencia sexual o física pueden ser parte o resultado del deterioro de la relación de pareja - como lo explican Heru, Stuart y Recupero (2007) - , en donde se afectan, como consecuencia de la interacción de numerosos factores, y entre otros elementos, las habilidades comunicativas. Teniendo esto en cuenta, la comunicación clara y directa es vista como una fortaleza de la pareja (Heru et al., 2007) y como un componente importante en la resolución no violenta de problemas (Devries et al. 2011; Espinoza-Bravo, 2018).

Adicional a esto, las mujeres ubicadas en el quintil superior de riqueza presentaron menos posibilidades de sufrir violencia sexual por parte de su pareja, lo cual resulta, asimismo, en un factor protector. En este aspecto, en un estudio en República Dominicana se reportó una relación inversa entre la violencia de pareja y el quintil de riqueza al que pertenece la muestra (Bott et al., 2013), sin embargo, los autores subrayaron en su informe sobre la violencia contra la mujer en América Latina y el Caribe la existencia de resultados ambiguos sobre esta asociación, y añadieron que las probabilidades de violencia no siempre son más bajas en las mujeres más ricas o instruidas. Para una mayor comprensión de estas diferencias se debería considerar el quintil de riqueza como un indicador del bienestar familiar y no necesariamente de la autonomía económica de la mujer, y, por ello, podría tener menor fuerza predictiva respecto a los ingresos de esta. En este sentido, la asociación entre violencia sexual de pareja e ingresos de la mujer no sería lineal y estaría mediada por otros factores contextuales (Ismayilova, 2015), y, quizás, en Perú, el empleo de una mujer podría representar un desafío a la expectativa cultural de que el hombre debe ser el único sostén familiar (Nóblega, 2012).

Otro aspecto importante es que con los modelos estadísticos se evidencia la interconexión de los niveles ecológicos, donde los efectos de algunos factores disminuyen y otros permanecen inalterados. Al respecto, el modelo ecológico de Heise (1998) propone que los factores se refuerzan y modifican recíprocamente (OMS, 2002), y en este sentido se encontró que el efecto predictor de los antecedentes de violencia familiar fue atenuado por el modelo conjunto, en el cual se solapan los niveles. Esta disminución puede deberse a la interacción con otros factores del microsistema - como la comunicación de pareja - y del exosistema - como el quintil de riqueza-. Asimismo, también se encontró que los factores del microsistema no modifican su efecto, lo que puede deberse a que estos operarían con cierta autonomía respecto a los demás niveles analizados. De este modo, aunque se postule la acción simultánea de los niveles y su recíproca implicación en la comprensión de la violencia (OMS, 2002; Flake, 2005; Puente-Martínez et al., 2016), considerando las diferencias descritas esta acción, la misma no sería paritaria en cuanto al peso predictivo de los factores en los niveles ecológicos.

Por último, el análisis del AUC dio cuenta de diferencias en la capacidad discriminante de los modelos, con lo que se demostró que los modelos dos y cuatro presentaron buena capacidad discriminatoria de la violencia sexual reciente. Una explicación para esto se relaciona con la elección de las variables predictoras a partir del modelo ecológico de Heise (1998), pues con este se considera la interacción entre factores personales, de pareja y sociales que la impactan; y como el Modelo 4 contiene todos los factores, fue este mismo el que presentó mayor capacidad discriminante. Eso último da soporte a la tesis de Bronfenbrenner (1977) con respecto al hecho de considerar la ecología entera del individuo.

Entre las limitaciones del estudio, es importante señalar que resultó imposible obtener información sobre otros predictores de la violencia sexual de pareja, pues la metodología del estudio consistió en un análisis secundario de datos. Por ejemplo, a nivel del macrosistema, no se exploraron variables como el aislamiento de la mujer y la familia, lo que limita la consideración complexiva del modelo ecológico. Asimismo, se contó solamente con información de la mujer acerca de su pareja, y en este aspecto el sesgo del recuerdo pudo haber conducido a una subestimación de las asociaciones entre la violencia sexual reciente y los episodios pasados de violencia. Sin embargo, en el análisis destaca el haber contado con una muestra nacional representativa de mujeres peruanas que, adicionalmente, delineó un perfil de las víctimas de violencia sexual; incluso, se realizó un análisis robusto que permitió considerar la especificidad de los modelos estadísticos en la violencia sexual.

En conclusión, desde la perspectiva ecológica, los factores que aumentan el riesgo a experimentar violencia sexual en nivel personal son haber presenciado violencia del padre hacia la madre y haber sido víctima de violencia física por parte del padre; mientras que en el microsistema figuran la violencia física — siendo esta un predictor bastante fuerte-, las amenazas de tipo económico y la 
frecuencia de embriaguez de la pareja. Por otra parte, la adecuada comunicación y pertenecer a quintiles superiores de riqueza actúan como protectores frente a la violencia sexual de pareja; y se evidencia que la interconexión entre los niveles ecológicos influye sobre el efecto de los factores en los modelos empleados. De este modo, el enfoque ecológico explica heurísticamente la violencia a través de la interacción simultánea y recíproca entre los niveles, aunque se debe añadir que esta interacción puede no ser paritaria, ya que existen diferencias en el poder predictivo de los factores.

Finalmente, los hallazgos de esta investigación sugieren realizar actividades de prevención primaria de la violencia sexual con énfasis en acciones a nivel del microsistema, pues las intervenciones que refuercen las habilidades comunicativas de las parejas resultan de gran importancia para potenciar su influencia protectora. Por otra parte, se precisa realizar actividades de prevención primaria con parejas que presenten varios factores de riesgo, como lo son el ser convivientes, pertenecer a quintiles inferiores en términos de riqueza, residir en áreas rurales, o que hayan reportado violencia física por parte del compañero. Y, por último, se recomienda realizar más investigación sobre el solapamiento entre la violencia económica y la violencia sexual. Ulteriores estudios son necesarios para lograr la comprensión de la interacción no paritaria de los niveles ecológicos con el fin de explicar a profundidad el fenómeno multicausal de la violencia sexual de pareja en el contexto peruano.

\section{Referencias}

Abramsky, T., Watts, C. H., Garcia-Moreno, C., Devries, K., Kiss, L., ... Heise, L. (2011). What factors are associated with recent intimate partner violence? Findings from the WHO multi-country study on women's health and domestic violence. BMC Public Health, 11(1), 1471-2458. doi: https://doi.org/10.1186/1471-2458-11-109

Alarcón, L., \& Ortiz, J. (2017). Qué factores se asocian a la violencia psicológica doméstica? Análisis del último decenio de la ENDES. Casus Revista de investigación y casos en salud, 2(1), 11-27. Recuperado de http://casus.ucss.edu.pe/ index.php/casus/article/view/28/12

Aldarondo, E., Kantor, G. K., \& Jasinski, J. L. (2002). A risk marker analysis of wife assault in Latino families. Violence Against Women, 8(4), 429-454. doi: https://doi. org/10.1177/107780120200800403

Bandura, A. (1977). Social Learning Theory. Englewood Cliffs: Prentice Hall.

Barría-Muñoz, J. R., \& Macchiavello-Rodríguez, A. (2012). Anatomía de los hombres que ejercen violencia hacia sus parejas: primer levantamiento de datos para el diseño de un perfil. Psicologia.com [Internet], 16(19), 1-29. Recuperado de https://www.academia.edu/27419754/Anatomia_de los_hombres_que_ejercen_violencia_hacia_sus_parejas

Barrios, Y. V., Gelaye, B., Zhong, Q., Nicolaidis, C., Rondon, M. B., Garcia, P. J., \& Williams, M. A. (2015). Association of childhood physical and sexual abuse with intimate partner violence, poor general health and depressive symptoms among pregnant women. PloS one, 10(1), e0116609. doi: https://doi.org/10.1371/journal.pone.0116609

Blitchtein, D., \& Reyes, E. (2012). Factores asociados a violencia física reciente de pareja hacia la mujer en el Perú, 2004-2007. Revista Peruana de Medicina Experimental y Salud Pública, 29(1), 35-43. Recuperado de http://www. scielo.org.pe/pdf/rins/v29n1/a06v29n1.pdf

Bott, S., Guedes, A., Goodwin, M., \& Mendoza, J. A. (2013). Violencia contra la mujer en América Latina y el Caribe: análisis comparativos de datos de 12 países. Washington: OPS.

Bronfenbrenner, U. (1977). Toward an experimental ecology of human development. The American Psychologist, 32(7), 513531. doi: http://dx.doi.org/10.1037/0003-066X.32.7.513

Casique, I. (2010). Factores de empoderamiento y protección de las mujeres contra la violencia. Revista mexicana de sociología, 72(1), 37-71. Recuperado de http://www.scielo. org.mx/pdf/rms/v72n1/v72n1a2.pdf

Castillo, M. (2011). Violencia de pareja en el Paraguay según la Encuesta Nacional de Demografía y Salud Sexual y Reproductiva 2008. Revista Latinoamericana de Población, 5(9), 27-48. Recuperado de https://www.redalyc.org/ pdf/3238/323827305002.pdf

Castro, R. J., Cerellino, L. P., \& Rivera, R. (2017). Risk factors of violence against women in Peru. Journal of family violence, 32(8), 807-815. doi: https://doi.org/10.1007/ s10896-017-9929-0.

Devries, K., Watts, C., Yoshihama, M., Kiss, L., Schraiber, L. B., ... Garcia-Moreno, C. (2011). Violence against women is strongly associated with suicide attempts: evidence from the WHO multi-country study on women's health and domestic violence against women. Social science \& medicine, 73(1), 79-86. doi: https://doi.org/10.1016/j. socscimed.2011.05.006

Ellsberg, M. C., Winkvist, A., Peña, R., \& Stenlund, H. (2001). Women's strategic responses to violence in Nicaragua. Journal of Epidemiology \& Community Health, 55(8), 547555. doi: http://dx.doi.org/10.1136/jech.55.8.547

Enríquez-Canto, Y., Ortiz-Romaní, K., \& Ortiz-Montalvo, Y. (2017). Análisis de los determinantes próximos e impacto de la ocupación en la fertilidad de mujeres peruanas. Revista Panamericana de Salud Pública, 41(1), 1-10. Recuperado de https://www.scielosp.org/scielo.php?pid=S1020-49892017 000100208\&script $=$ sci_arttext\&tlng $=\mathrm{pt}$

Espelt A., Marí-Dell'Olmo M., Penelo E., Bosque-Prous M. (2017). Estimación de la Razón de Prevalencia con distintos 
modelos de Regresión: Ejemplo de un estudio internacional en investigación de las adicciones. Adicciones, 29(2), 105112. doi: https://doi.org/10.20882/adicciones. 823

Espinoza-Bravo, S. P. (2018). Violencia sexual en la pareja: una práctica invisibilizada (tesis de licenciatura), Universidad Academia de Humanismo Cristiano, Santiago de Chile, Chile. Recuperado de http://bibliotecadigital.academia.cl/ handle/123456789/4601

Flake, D. F. (2005). Individual, family, and community risk markers for domestic violence in Peru. Violence against women, 11(3), 353-373. doi: https://doi. org/10.1177/1077801204272129

Garcia-Moreno, C., Jansen, H., Ellsberg, M., Heise, L., \& Watts, C. (2006). Prevalence of intimate partner violence: findings from the WHO multi-country study on women's health anddomestic violence. Lancet, 368(9543), 12601269. doi: https://doi.org/10.1016/S0140-6736(06)69523-8

Halford, W. K., Sanders, M. R, \& Behrens, B. C. (2000). Repeating the errors of our parents? Family-of-origin spouse violence and observed conflict management in engaged couples. Family Process, 39(2), 219-235. doi: https://doi. org/10.1111/j.1545-5300.2000.39206.x

Heise, L. L. (1998). Violence against women: An integrated, ecological framework. Violence Against Women, 4(3), 262290. doi: https://doi.org/10.1177/1077801298004003002

Heru, A. M., Stuart, G. L., \& Recupero, P. R. (2007). Family functioning in suicidal inpatients with intimate partner violence. Primary care companion to the Journal of clinical psychiatry, 9(6), 413-418. Recuperado de https://www.ncbi. nlm.nih.gov/pmc/articles/PMC2139922/

Instituto Nacional de Estadística e Informática [INEGI]. (2017a). Encuesta Demográfica y de Salud familiar ENDES-2017. Nacional y Departamental. Recuperado de https://www.inei.gob.pe/media/MenuRecursivo/publica ciones_digitales/Est/Lib1525/index.html

Instituto Nacional de Estadística e Informática [INEGI]. (2017b). Perú: Indicadores de violencia familiar y sexual, 2000-2017. Recuperado de https://www.inei.gob.pe/media/ MenuRecursivo/publicaciones_digitales/Est/Lib1465/libro. pdf

Ismayilova, L. (2015). Spousal violence in 5 transitional countries: A population-based multilevel analysis of individual and contextual factors. American journal of public health, 105(11), e12-e22. doi: https://doi.org/10.2105/ AJPH.2015.302779

Jaén, C., Rivera, S., Amorin, E., \& Rivera, L. (2015). Violencia de Pareja en Mujeres: Prevalencia y Factores Asociados. Acta de Investigación Psicológica, 5(3), 2224-2240. doi: http://dx.doi.org/10.1016/s2007-4719(16)30012-6

Karamagi, C. A., Tumwine, J. K., Tylleskar, T., \& Heggenhougen, K. (2006). Intimate partner violence against women in eastern Uganda: implications for HIV prevention. BMC public health, 6(1), 284. doi: https://doi. org/10.1186/1471-2458-6-284
Kishor, S., \& Kiersten, J. (2006). Perfil de la violencia doméstica: un estudio en varios países. Calverton: Macro International Inc. Recuperado de https://dhsprogram.com/ pubs/pdf/OD38/OD38.pdf

Krebs, C., Breiding, M. J., Browne, A., \& Warner, T. (2011). The association between different types of intimate partner violence experienced by women. Journal of Family Violence, 26(6), 487-500. doi: https://doi.org/10.1007/ s10896-011-9383-3

Martínez, A., Landa, S., Echeburúa, E., \& Rovira, D. (2016). Factores de riesgo asociados a la violencia sufrida por la mujer en la pareja: una revisión de meta- análisis y estudios recientes. Anales de Psicología, 32(1), 295-306. doi: http:// dx.doi.org/10.6018/analesps.32.1.189161

Montero, I., \& León, O. G. (2007). A guide for naming research studies in Psychology. International Journal of Clinical and Health Psychology, 7(3), 847-862. Recuperado de http:// www.aepc.es/ijchp/GNEIP07_es.pdf

Nóblega, M. N. (2012). Risk and protective factors for physical and emotional intimate partner violence against women in a community of Lima, Peru. Journal of interpersonal violence, 27(18), 3644-3659. doi: https://doi. org/10.1177/0886260512447522

Organización Mundial de la Salud [OMS]. (2002). Informe mundial sobre la violencia y la salud. Washington, D. C.: OMS. Recuperado de https://www.who.int/vio lence_injury_prevention/violence/world_report/es/ summary_es.pdf

Organización Mundial de la Salud [OMS]. (2005). Estudio multipaís de la OMS sobre salud de la mujer y la violencia doméstica: resumen del informe. Ginebra: OMS. Recuperado de http://www.who.int/iris/handle/10665/43390

Organización Mundial de la Salud [OMS]. (2012). Understanding and addressing violence against women: Intimate partner violence (Paper No. WHO/RHR/12.36). Ginebra: OMS. Recuperado de https://apps.who.int/iris/ handle/10665/77432

Organización Mundial de la Salud [OMS]. (2013). Global and regional estimates of violence against women: prevalence and health effects of intimate partner violence and nonpartner sexual violence. Washington, D. C.: OMS.

Postmus, J. L., Plummer, S. B., McMahon, S., Murshid, N. S., $\&$ Kim, M. S. (2012). Understanding economic abuse in the lives of survivors. Journal of Interpersonal Violence, 27(3), 411-430. doi: https://doi.org/10.1177/0886260511421669

Puente-Martínez, A., Ubillos-Landa, S., Echeburúa, E., \& Páez-Rovira, D. (2016). Factores de riesgo asociados a la violencia sufrida por la mujer en la pareja: una revisión de meta-análisis y estudios recientes. Anales de psicología, 32(1), 295-306. doi: http://dx.doi.org/10.6018/ analesps.32.1.189161

Rey-Anacona, C. A. (2017). Diferencias por sexo y variables asociadas con las agresiones sexuales en el noviazgo en universitarios. Psychologia. Avances de la disciplina, 
11(1), 25-37. Recuperado de https://www.redalyc.org/ pdf/2972/297251403002.pdf

Rodriguez-Menés, J., Puig, D., \& Sobrino, C. (2014). Polyand distinct-victimization in histories of violence against women. Journal of Family Violence, 29(8), 849-858. doi: http://dx.doi.org/10.1007/s10896-014-9638-x

Stark, E. (2007). Coercive control: How men entrap women in personal life. Nueva York, EE. UU.: Oxford University Press. 